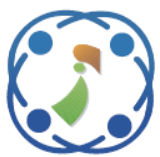

\title{
Performance Modeling of Bio-Inspired Routing Protocols in Cognitive Radio Ad Hoc Network to Reduce End-to-End Delay
}

\author{
Ramkumar Jaganathan $^{1 *} \quad$ Vadivel Ramasamy $^{2}$ \\ ${ }^{1}$ Department of Computer Science, VLB Janakiammal College of Arts and Science, India \\ ${ }^{2}$ Department of Information Technology, Bharathiar University, India \\ * Corresponding author's Email: jramkumar1986@gmail.com
}

\begin{abstract}
The rapid advancement of Cognitive Radio Ad Hoc Network (CRAHN) has created a demand for effectively utilizing the spectrum without affecting the environment. End-to-end delay in CRAHN affects the performance in numerous ways. The major task of routing protocols is to transfer the data to the destination in a shorter duration by utilizing low energy. Congestion in the network leads a way to end-to-end delay and exhaustive energy consumption, but optimization can be used to control it. This research work aims to propose a bio-inspired routing protocol inspired from characteristics from wolves to reduce the overall end-to-end delay which results in reducing the consumption of energy and extends the lifetime of the network. This research work utilizes M/G/1 queueing model to avoid network congestion, where the requests from nodes are markovian and service times regarding the requests have general distribution time. With benchmark performance metrics NS2 simulations are carried out to check the performance of the proposed protocols. Results conclude that the proposed protocol significantly outperforms than other protocols with better packet delivery ratio, reduced end-to-end latency and energy consumption.
\end{abstract}

Keywords: Cognitive radio ad hoc network (CRAHN), Bio-inspired, End-to-end delay, Routing, Protocol.

\section{Introduction}

To clear up the spectrum inadequacy drawback in the wireless communication system, Cognitive Radio (CR) technology has been proposed to the world. The users of CR technology can utilize the existent wireless spectrum strategically without disturbing or be impacting the licensed users. The networks that are enabled with CR devices provide an associate degree level benefit in wireless communications. Moreover, $\mathrm{CR}$ is a radio-based technology which can change its transmitter parameters in the community with surroundings, wherein it firmly performs. In controlling a CR in a straightforward way, it will indisputably be software oriented, where the codings are written after well understanding the essentials of CR. CR is an embodiment design of remote networking that senses its surroundings, trace the updating and commonly barter message with their networks [1].
Adding this feature of understanding the network of ad hoc by a glance to the geo-location based network is known as cognitive radio ad-hoc network (CRAHNs). CRAHN aims to improve the way of radio spectrum is utilized.

The four basic functions [2] of CR networks are listed below:

- Spectrum Sensing: identification of all available spectrum channels in order to avoid interference.

- Spectrum Sharing: exchange information related to spectrum availability between neighbor nodes.

- Spectrum Mobility: provide faultless connectivity between nodes.

- Spectrum Decision: detects the best available vacant spectrum channels from detecting spectrum holes. 
CRAHNs are autonomous networks enabled with CR technology, where it does not need the support of base stations or any other infrastructure based support [3]. Devices used in CRAHN are based on the battery that they hold. The present technologies used in the battery will make it work for a long period of time with the tolerable energy, even when it is charged for a shorter duration. One of the very important issues of CRAHN is the conservation of energy because the batteries hold the limited capacity of energy. The nodes of CRAHN get dead when the battery life gets complete. The two things that determine the duration of nodes are conservation of energy and battery life.

There exist two kinds of users in CRAHN, which are Primary User (PU) and Secondary User (SU). PU's are authorized to access the network at an associate degree level because they hold the license. Normally these users are given the maximum preference to use the spectrum and are liable to assured quality-of-service (QoS). SU's are authorized to access the network but not in an associate degree level as like the PU. Preferences are not given much to these users and QoS are also not maintained as like PU. Primitive concept of CR network is to move out the SU (which don't have any license) from the network when PU (which has a license) is spotted [3]. The opportunity of using the CRAHN is unstable for the SU with time and location, due to the importance is given to PU. In this manner, routing of data packets in CRAHNs requires cooperation between its module and functionality of spectrum management for continuous aware of the physical environment to make more accurate decisions [4, 5]. Exhaustive usage of spectrum by PU will give way for routing problem in CRAHNs. For this reason, it's always necessary to design a routing protocol that will effectively allocate the spectrum for the available users regarding their utilization.

A network is a place where two or more communicating devices involve for the exchange of data with the principle of a store and forward. The network is categorized into two types which are wire-based network and wireless-based network. A wireless-based network is further classified into (a) infrastructure-based wireless network; and (b) infrastructure-less wireless network. There will not be any occurrence of any critical effect in the topology of the network even if the battery energy goes down in an infrastructure based wireless network. But it is inversely proportional in the case of the infrastructure-less wireless network; the reason is each node in the network is acting as a sequence of a source, destination as well as the router also. The routing gets affected when a node gets dead due to battery down, at the point, the network topology gets updated and sometimes network is segregated.

\subsection{Problem definition}

Currently, CRAHN is facing enormous energy loss due to ineffective utilization of nodes. This is due to not considering the preservation of energy in the design of routing protocols. Energy consumption gets increased when the distance of the destination gets increased. Ordinary traditional protocols get suits for many ad hoc networks, but it will never support CRAHN due to geographic area coverage. Without knowing this information many protocols are being proposed for CRAHN, resulting in network failure and increased end-to-end delay. Also, avoidance of congestion leads to a successful network. Solving the congestion at root level and passing the congestion information around the network will improve the performance of CRAHN at various aspects.

\subsection{Motivation and objectives}

The existing protocols of CRAHN are suffering by enormous energy consumption and end-to-end delay due to poor selection of routing path for data packet towards destination. Congestion is considered as an important reason for enormous energy consumption and end-to-end delay. If congestion is not considered or treated in an appropriate time, it will lead to network failure. In this paper, we propose a new bio-inspired routing protocol based on behavior and character of wolf. The proposed protocol reduces the energy consumption and end-to-end delay sharing the congestion information to the neighbor nodes. That is, the proposed protocol does not share the congestion information with the nearby neighbors only, but with the entire network. M/G/1 queuing model is used to avoid congestion rather than firstin-first-out model, which results in increase in network performance. Further, proposed protocol works well even in heterogeneous environment and the results are better when comparing with the existing method IFLIP [16] and TIGHT [17].

The remainder of this paper is organized as follows. Section 2 provides the brief of the related works as literature review. Section 3 discusses the proposed protocol Wolf Prey Inspired Protocol (WPIP) for CRAHN. Section 4 illustrates the chosen performance metrics along with the NS2 simulation settings. Section 5 confers the results. Finally, 
Section 6 concludes the paper with future dimensions.

\section{Literature review}

Energy Consumption [6] problem was studied under the constraint of probabilistic interference to primary receivers and proposed a centralized solution for optimal spectrum assignment cum route selection. But the proposed method didn't meet the optimum level of energy consumption. Transport capacity of cooperative CRAHN was analyzed and a half-slotted ALOHA multiple access protocols [7] was proposed to show the increase in primary coverage radius, but the results showed that it consumed more energy which cannot be accepted. Cognitive radio routing protocol [8] was proposed to reduce the routing problems that arise in CRAHN, but the solutions provided for routing problems results in finding the lengthier route which consume more delay and energy. Hybrid firefly simulated annealing based spectrum opportunistic routing algorithm [9] was proposed to address issues in spectrum mapping in local and global spectrum sensing information, but the results gone down in sensing the global information.

Spectrum aware cross-layer medium access control protocol [10] was proposed for CRAHN with the concept of splitting the network into clusters. Cluster information was defined as maximum edge biclique problem. When the cluster level gets increased, there exist degrade in overall network performance. Joint rate adaptation, channel assignment and routing approach [11] was proposed to maximize social welfare by optimizing the resource utility in multi-channel multi-hop CRAHNs, where the routing mechanism has just considered relay workload, distance, and co-channel interference to primary and secondary nodes. The results demonstrated that it's necessary to consider the bench mark metrics and the not the ordinary metric for good performance. The problems in spectrum-aware routing in a multi-hop were studied and a distributed strategy learning mechanism [12] was proposed based on stochastic fictitious play. It aimed to give enhanced performance in limited information exchange and potential routing-towardprimary attacks, where the result lacks in security attacks.

Delay-minimized routing protocol [13] proposed for multichannel multi-flow mobile cognitive ad hoc networks predicts the delay based on conflict probability. Most times the predicted delay was much higher than the actual delay and this will lead to network failure. Multipath algorithm [14] was proposed to reduce the interference of information. It utilizes the more reliable routing approach to identify the suitable links for different data transmission, where the result shows that the multipath algorithm sometime found the poor quality path for high capacity data packet. Energyefficient and reliable cognitive ad-hoc routing protocol [15] was proposed to provide reliable transmission path and prolong network lifetime in cognitive radio networks. The result demonstrate that there exist an increase in packet loss probability, link latency and residual energy for path establishment.

A bio-inspired based approach, namely IFLIP [16] was made to solve the issues of routing in CRAHN. In this approach routing paths were optimized by utilizing leaping behavior of frog, It results in reduced energy consumption and end-toend delay, which was due to avoiding the congestion. Geographic routing protocol named TIGHT [17] was presented to increase the transmission opportunities of users in CRAHN. Greedy approach was used to increase the opportunities, where it didn't considered the importance of other users. Due to this approach the protocol may succeed in results for specific users which lead to network failure.

\section{Wolf prey inspired protocol (WPIP)}

The need for bio-inspired algorithms gets increased day by day due to its nature of applicability in social welfare, where the heuristic algorithms nature is to apply for a specific problem. Optimization is the process of effectively utilizing the situation or resource for the increased positive result. Purpose of utilizing the bio-inspired algorithms is to utilize the concepts of nature to solve the issues that arise in the current world. Grey wolf optimizer [18] algorithm inherited from the social hierarchy and hunting behavior of grey wolves, which comes under Canidae family. Generally, the wolves live in groups. Group is wolves are called as packs. A pack may contain up to 7 to 8 wolves. The communication method used by the wolves use seems to be very essential for an endurance of pack. Each and every member of the pack, work in a cooperative manner for hunting and keeping territory safe in order to reach the target successfully.

\subsection{Wolves and its types}

In general, animals are categorized into 3 major types based on its food habitats. They are herbivorous, carnivorous, and omnivorous. In this, 
Table 1. Key actions of members

\begin{tabular}{|c|l|}
\hline Members & \multicolumn{1}{c|}{ Key Actions } \\
\hline$\alpha$ & $\begin{array}{l}\text { Leaders of a pack, which has been led by both male and female wolves. Its role is to decide several } \\
\text { activities like hunt, shelter, and schedule. }\end{array}$ \\
\hline$\beta$ & Associate leaders, which assist $\alpha$ while making decisions. \\
\hline$\delta$ & Dominators, which dominates $\gamma$ and $\omega$ to complete the target. \\
\hline$\gamma$ & Observers, it observes every movement that occurs in and around hunting area. \\
\hline$\omega$ & Information Conveyors to all other wolves. \\
\hline
\end{tabular}

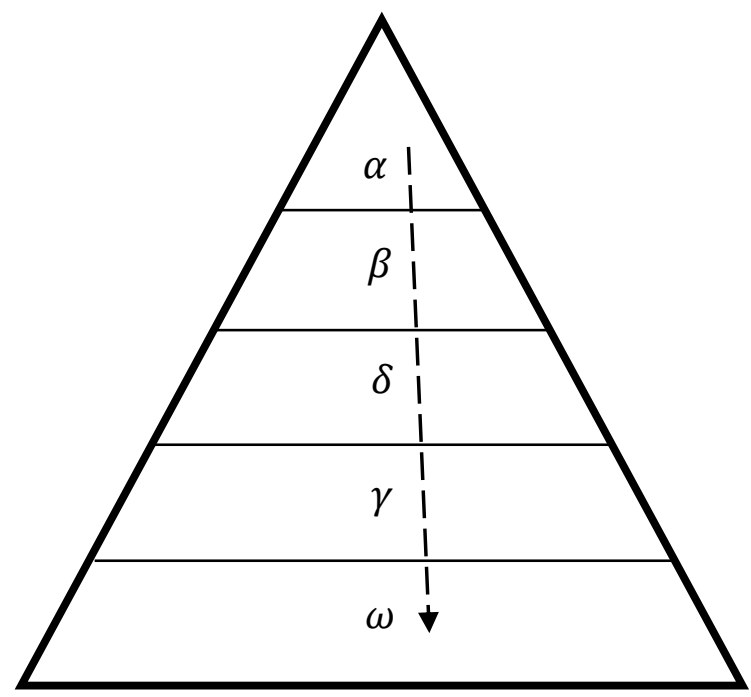

Figure. 1 Structure of commanding

wolves are from carnivorous family. They consume flesh by hunting its prey. There exists a lot of predators, among them wolves are very special because of its hunting strategy and bonding with its pack. Wolves species were classified into 7 groups based on their physical appearance and geological surviving regions, which are (i) Grey Wolf (ii) Arctic Wolf (iii) Red Wolf (iv) Indian Wolf (v) Himalayan Wolf (vi) Ethiopian Wolf, and (vii) Eastern Wolf. Even though wolves are classified into several groups, all of them have the same characteristics and hunting strategy because of its inherited behavior from the ancestral species grey wolves.

\subsection{Key actions}

Wolves are successful hunters in predator family. They plan their pack towards routine activity. Along with this, several actions take place within the pack, which are (i) target selection, (ii) encircling (iii) hunting, (iv) attacking, and (v) searching. The above-mentioned actions were done by members of the pack based on their maturity and hunting experience. The members of the pack were technically termed as alpha $(\alpha)$, beta $(\beta), \operatorname{delta}(\delta)$, gamma $(\gamma)$, and omega $(\omega)$. These terms are applicable to all the above-mentioned wolves species. The key actions of members of the pack are given in Table 1, and commanding structure of members are given in Fig. 1.

\subsection{Key actions}

With a specific end goal to scientifically show the commanding structure of wolves in WPIP, we consider $\alpha$ as the fittest solution. $\beta, \delta$ and $\gamma$ as the second, third and fourth best solution. $\omega$ is considered as candidate solution excluding $\alpha, \beta, \delta$, and $\gamma$. In WPIP, the hunting for prey is directed by $\alpha, \beta, \delta$, and $\gamma$, where the $\omega$ wolves obey and follow other members commands in the pack.

\subsection{Target selection}

In prey hunt, wolves core job is to select the target, which is done by $\alpha$. The target is selected based on the number of members in the pack, because the state of affairs may change during the hunt, (i.e., the target may change).

\subsection{Encircling for prey}

Once after the command received from $\alpha$, the next step of encircling the prey will be processed by $\beta$ for hunting. With a specific end goal to scientifically show the wolves behavior in encircling 
to hunt for prey, following equations (Eq. (1) and Eq. (2)) is used:

$$
\begin{gathered}
\vec{D}=\left|\vec{C} \cdot \overrightarrow{X_{P}}(t)-\vec{X}(t)\right| \\
\vec{X}(t+1)=\overrightarrow{X_{P}}(t)-\vec{A} \cdot \vec{D}
\end{gathered}
$$

where $t$ denotes the iteration number, $\vec{A}$ and $\vec{C}$ denotes the coefficient of position, $\overrightarrow{X_{P}}$ denotes the position of prey, $\vec{X}$ denotes the current position of grey wolf lf, $\vec{D}$ used to predict new or next position of grey wolf towards hunting for prey. $\vec{A}$ and $\vec{C}$ are calculated by utilizing[18]:

$$
\begin{gathered}
\vec{A}=2 \vec{a} \cdot \overrightarrow{r_{1}}-\vec{a} \\
\vec{C}=2 \cdot \overrightarrow{r_{2}}
\end{gathered}
$$

where the elements of $\vec{a}$ are precisely depreciated to 0 from 2 after certain iterations and $\overrightarrow{r_{1}}$ and $\overrightarrow{r_{2}}$ are random vectors 0 and 1 .

Eqs. (3) and (4) together exemplify the impact of Eqs. (1) and (2) in a two-dimensional figure with its position vector and few of feasible neighbors in the process of hunting. The grey wolf can revise its current position (prey, wolves) based on the position of prey (prey*, wolves*). Grey wolf's next better positions towards hunt for prey are calculated based on the current position by modifying the estimated value of $\vec{A}$ and $\vec{C}$ vectors. $\overrightarrow{r_{1}}$ and $\overrightarrow{r_{2}}$ are random vectors which allow the wolves to achieve a new position. Grey wolf can revise its current position close to the position of prey in any random using Eqs. (1) and (2). A similar idea can be used to seek a new position in $n$ dimension, where the wolves will move in hyper-cubes regarding the best solution.

\subsection{Hunting for prey}

Grey wolves can perceive the location of prey and encircle them. $\alpha$ generally guide the process of hunting. $\beta, \delta$, and $\gamma$ may likewise take an interest in hunting periodically. Nevertheless, in exploring the hypothetical space, wolves have no clue about the location of prey. With a specific end goal to mathematically show the hunting process of grey wolves, it is assumed that $\alpha, \beta, \delta$, and $\gamma$ have good knowledge about the next possible location of prey. The positions of $\alpha, \beta, \delta$, and $\gamma$ are updated to $\omega$ by using Eq. (5):

$$
\vec{X}(t+1)=\frac{X_{1}+X_{2}+X_{3}+X_{4}}{4}
$$

\subsection{Attacking for prey}

The hunting process is completed carried by grey wolves, once when the attack process gets complete. By default, the attack process gets complete when the prey stops moving from its current position. To mathematically show the move of grey wolves towards prey, it's necessary to reduce the value of $\vec{a}$, that is, $\vec{A}$ is a random value between $-4 a$ and $4 a$ throughout the iterations. When $\vec{A}$ is a random value between -1 and 1 , the grey wolves next position may be in between its current position and prey position. By this stage, positions of other wolves are updated based on grey wolves position.

\subsection{Searching for prey}

Based on the position of $\alpha$, searching is done by $\beta$ and $\delta$ wolves for the prey hunt, but there exists a chance for deviation to search for prey and concentration to attack prey. To show mathematically, grey wolves deviation in search towards prey, when applying a random value that is either greater than 1 or less than -1 to $\vec{A}$. $\vec{C}$ holds random values between 0 and 3 , where it applies random value to prey to stochastically increase or decrease prey vision of defining the distance from its current position.

\section{9 $M / G / 1$ queue model}

This research utilizes the $M / G / 1$ [19] queueing model to avoid congestion in CRAHN. It is possible to receive the distribution of the waiting time by the following. Let $N_{k}$ denote multiple nodes that are waiting behind by the $K^{\text {th }}$ node that is getting serviced from the server, and let $A_{k}$ denotes the mobile node request sent in random order which arrive the station while $K^{\text {th }}$ node is being serviced. Relationship between the node and server for the service are obtained by Eq. (6).

$$
N_{k+1}= \begin{cases}\sum N_{k}-1+A_{k+1} & N_{k}>0 \\ \sum A_{k+1} & N_{k}=0\end{cases}
$$

It is clearly evident that $\left\{N_{k}, k=0,1,2, \ldots\right\}$ forms the markov chain and it is termed as $M / G / 1$ [19] markov chain. 
Considering the transition probabilities, $M / G / 1$ markov chain can be stated as

$$
P_{i j}=P\left[N_{k+1}=1 \mid N_{k}=i\right]
$$

where the value of $\mathrm{N}_{\mathrm{k}}$ is always less than $N_{k+1}$, it has $P_{i j}=0$ for $j<i-1$. Thus, for $j>=(i-1)$, $P_{i j}$ is the probability of having $(j-i+1)$ nodes arrived arriving with more than 1 request while the servicing $(k+1)^{t h}$ node. When considering the $i>$ $0, P_{i j}$ denotes the probability of exact $j$ customers have arrived in the system while the $(k+1)^{t h}$ customer is being serviced.

Let the random variable $Z$ indicate the number of nodes that reach the system during the service amid. At that point the $P_{i j}$ is given by

$$
\begin{aligned}
& p_{Z}(n)=P[Z=n] \\
& =\int_{x=0}^{\infty} \frac{(\lambda x)^{n}}{n !} e^{-\lambda x} f_{x}(x) d x \quad n=0,1, \ldots
\end{aligned}
$$

While defining $\alpha_{\mathrm{n}}=\mathrm{P}[\mathrm{Z}=\mathrm{n}]$, then state transition matrix of Markov chain will be given as

$$
P=\left[\begin{array}{cccccc}
\alpha_{0} & \alpha_{1} & \alpha_{2} & \alpha_{3} & \cdots & \cdots \\
\alpha_{0} & \alpha_{1} & \alpha_{2} & \alpha_{3} & \cdots & \cdots \\
0 & \alpha_{0} & \alpha_{1} & \alpha_{2} & \cdots & \cdots \\
0 & 0 & \alpha_{0} & \alpha_{1} & \cdots & \cdots \\
0 & 0 & 0 & \alpha_{0} & \cdots & \cdots \\
\cdots & \cdots & \cdots & \cdots & \cdots & \cdots \\
\cdots & \cdots & \cdots & \ldots & \cdots & \ldots
\end{array}\right]
$$

Finally the transformation of $Z$ can be given as

$$
\mathrm{G}_{\mathrm{Z}}=\mathrm{M}_{\mathrm{x}}(\lambda-\lambda \mathrm{Z})
$$

Reason for arise of congestion in the CRAHN is due to excessive length of queue which results in increased waiting time in getting the service from the server. The main purpose of using $M / G / 1$ [19] queue in this research work is to avoid the waiting time of nodes for getting service/request, because the nodes waiting in the queue will know the average or expected waiting time. In case of time consideration, the nodes can move to the next server to process its request.

\section{Simulator}

This section discusses the evaluation of the proposed protocol WPIP using NS2 simulations. For CRAHN, there exist no generally available implementations regarding the protocols.
Table 2. Simulation settings and parameters

\begin{tabular}{|c|c|}
\hline Area Size & $4000 \times 3000 \mathrm{~m}^{2}$ \\
\hline No. of Primary User & 4 to 10 \\
\hline No. of Secondary User & 1200 to 1600 \\
\hline MAC & $802.11 \mathrm{~b}$ \\
\hline Transmission Range & 2500 meters \\
\hline Simulation Time & 500 seconds \\
\hline Traffic Source & CBR \\
\hline Packet Size & 512 bytes \\
\hline Total Packets & 8000 \\
\hline Mobility Model & Random Waypoint Model \\
\hline Initial Energy & 0.5 Joules \\
\hline
\end{tabular}

Additionally, the available details regarding the implementations are not clear to honestly understand the performance they give. This research work opted to make a comparison with IFLIP [16] and TIGHT [17] which sends packets over the secondary channel only. $\mathrm{C}++$ is chosen to write the simulation code and NS2 simulator is used for the simulation. The simulation result found that WPIP has similar packet delivery ratio (PDR) to that of TIGHT [17] in small-scale CRAHN. Considering end-to-end latency, WPIP outperforms IFLIP [16] and TIGHT [17]. It is a well-known fact that NS2 have poor performance in simulating large-scale networks, so this research work has opted for $\mathrm{C}++$ simulations to analyze the performance of WPIP in large-scale networks. Reliability of $\mathrm{C}++$ simulations is extremely high for all the protocols. Spontaneous network topology was used for routing the packets. Accordingly, the assessment of WPIP is done by comparing with IFLIP [16] and TIGHT [17]. For the assessment, data packets are generated over random network topologies and sent to the nodes in mobile. Table 2 demonstrates the simulation settings.

\section{Performance evaluation}

The benchmark metrics are used to measure the performance of WPIP against IFLIP [16] and TIGHT [17] protocols are Packet Delivery Ratio, Throughput, Energy Consumption, End-to-End Delay and Social Welfare.

- Packet Delivery Ratio: It is the measure of the number of packets successfully received by the destination and the total number of packets sent by the source.

- Throughput: It is the measure of the total amount of data transferred from source node to 


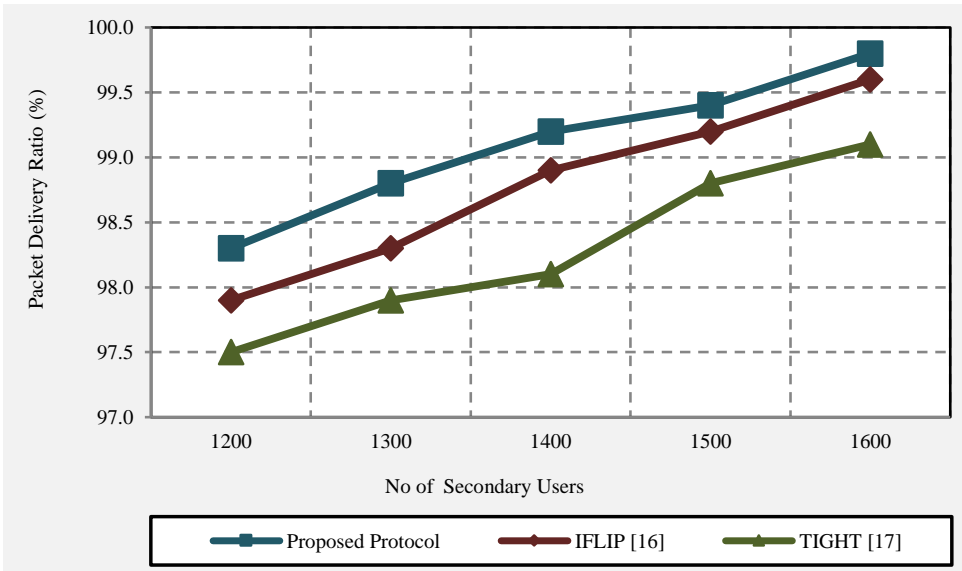

Figure. 2 Packet delivery analysis over secondary users

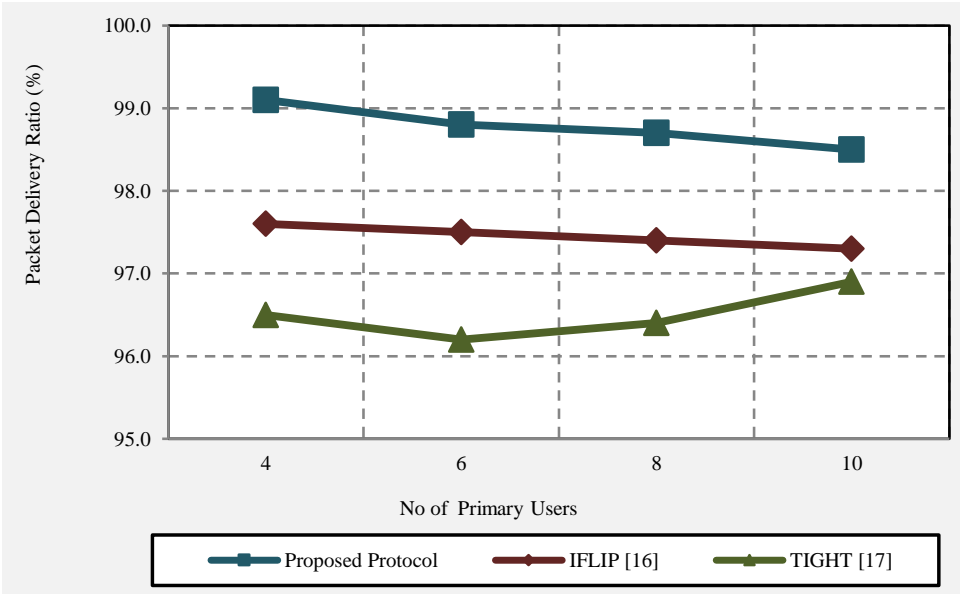

Figure. 3 Packet delivery analysis over primary users

destination node or processed in a specified amount of time.

- Energy Consumption: It is the measure of energy consumed by the data packet from source node to destination node.

- End-to-End Delay: It is the measure of time taken by a packet to transmit across a network from source to destination.

- Social Welfare: It is the measure of global efficiency in saving energy, the difference between energy saved node and actual energy required by the individual node to sustain the communication.

\subsection{Packet delivery ratio analysis}

In Fig. 2, secondary users varying from 1,200 to 1,600 are plotted in the $\mathrm{x}$-axis, where the $\mathrm{y}$-axis is plotted with packet delivery ratio. Fig. 2 compares the packet delivery ratio of WPIP with other protocols under a varying number of secondary users. In the simulation, all three protocols are made to send data packets over the auxiliary channel. The routing of the packet relies upon the unconstrained system topology. Assessment of the proposed protocol (WPIP), IFLIP [16] and TIGHT [17] are done under node mobility by sending packets over various randomly produced network topologies. It is due to utilization of wolf behavior in searching for the route to send the data. WPIP is designed to share the routing and congestion information to all the nodes around the network, where the other protocols were designed to share the information only with the nearby neighbors. IFLIP [16] and TIGHT [17], strictly stick with the route that were established once, but the proposed protocol never stick with single route and it tries to sends the data packet in multiple route to reach the destination with the aim of increasing the packet delivery ratio. Fig. 2 clearly shows that the proposed protocol (WPIP) have better performance in delivering data packets to the destination than IFLIP [16] and TIGHT [17], in the increased secondary user scenario.

In Fig. 3, primary users varying from 4 to 10 are plotted in the $\mathrm{x}$-axis, where the $\mathrm{y}$-axis is plotted with packet delivery ratio. Fig. 3 made a comparison of 


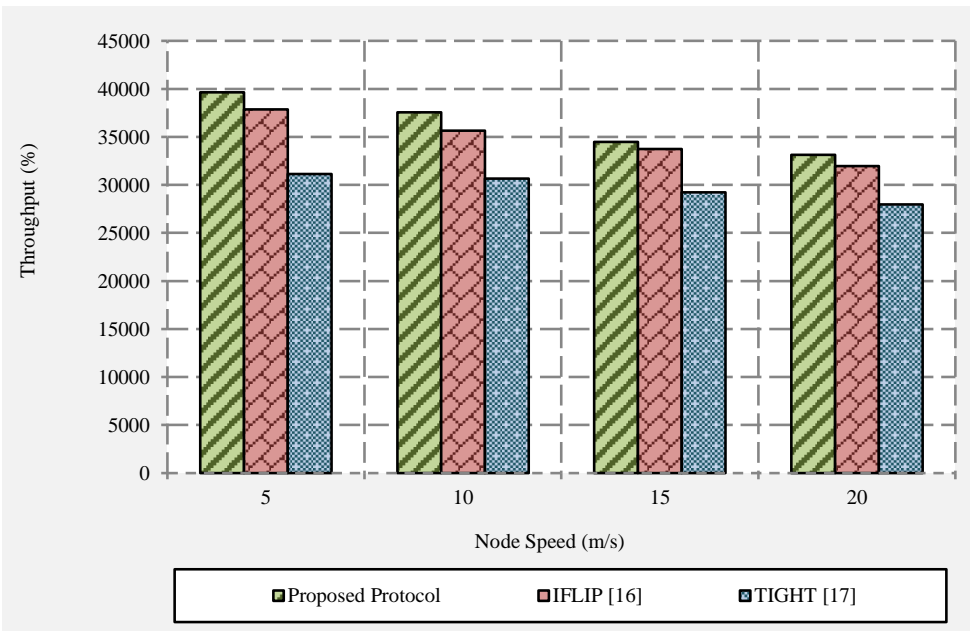

Figure. 4 Throughput analysis over mobility speed

packet delivery ratio of the proposed protocol (WPIP) with other protocols under varying number of primary users. In the simulation, all three protocols are made to send data packets over the auxiliary channel. It is possible to see that the packet delivery ratio of WPIP is sufficiently high when compared with IFLIP [16] and TIGHT [17] even with the increase of primary users. Some users may expect WPIP to give low packet delivery ratio when there is an increase in primary user, and also due to data packet taking lengthier time around the regions of primary users. Packet delivery ratio of the proposed protocol will never get decreased with the increase of primary users. The reasons behind this is, IFLIP [16] and TIGHT [17] were designed to utilize the auxiliary channels and it will never consider secondary channels even if it is available. Proposed protocol is designed to consider the secondary channels when it is available. By default, WPIP is designed to share the information with all the nodes in the network and not with nearby neighbors only. Fig. 3 clearly shows that the proposed protocol (WPIP) have better performance in delivering data packets to the destination than IFLIP [16] and TIGHT [17], in the primary user scenario.

\subsection{Throughput analysis}

In Fig. 4, the mobility speed ranging from 5 to 20 are plotted in the x-axis with the unit of interval 5, where the y-axis is plotted with throughput ranges from 0 to 45,000 with the unit of interval 5000. Fig. 4 compares the throughput of the proposed protocol (WPIP) with IFLIP [16] and TIGHT [17]. Proposed protocol (WPIP) gives better throughput than the others even there exist an increase in speed of the node. It is due to the design of WPIP in utilizing the multiple paths even it is lengthier than the ordinary route, where the other protocols stick with the route that were established once. Also it is to be noted that the proposed protocol too facing reduced throughput when there exist an increase in node speed. When analyzing the Fig. 4, it is evident the proposed protocol (WPIP) have better throughput in delivering data packets to the destination than IFLIP [16] and TIGHT [17], in the increased node speed scenario.

\subsection{Energy consumption analysis}

In Fig. 5, mobility speed ranges from 5 to 20 are plotted in the x-axis with the unit of interval 5, where the $y$-axis is plotted energy consumption ranging from 50 to 130 joules with the unit interval of 10. Fig. 5 compares energy consumed by WPIP to deliver the packet to the destination, with IFLIP [16] and TIGHT [17]. The WPIP routing protocol designed to select the most stable route toward the destination in the available multiple paths. The route selected by WPIP could be the best routing path and consumes less energy than other routes, with the shortest route, where the other protocols simply select the shortest path without checking its quality which results in route failure and retransmission. Due to route failure and retransmission enormous amount of energy is wasted by IFLIP [16] and TIGHT [17]. Fig. 5 clearly shows that the proposed protocol (WPIP) consumes less energy for delivering data packets to the destination than IFLIP [16] and TIGHT [17].

\subsection{End-to-end delay analysis}

In Fig. 6, bandwidth ratios varying from 2 to 11 are plotted in the $\mathrm{x}$-axis, where the y-axis is plotted with end-to-end delay varying from 0 to $42 \mathrm{~ms}$. Fig. 6 compares the end-to-end delay faced by the 


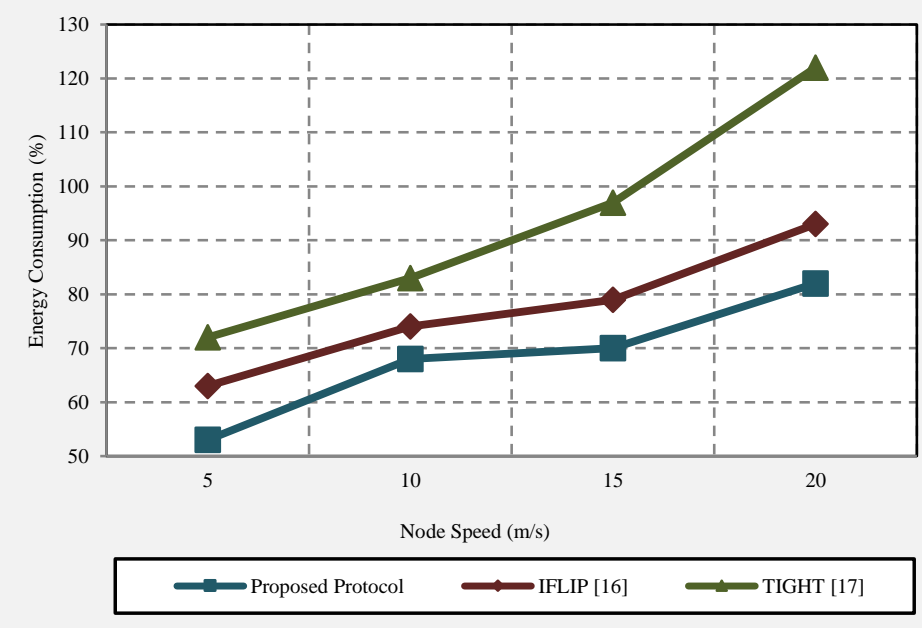

Figure. 5 Energy consumption over node speed

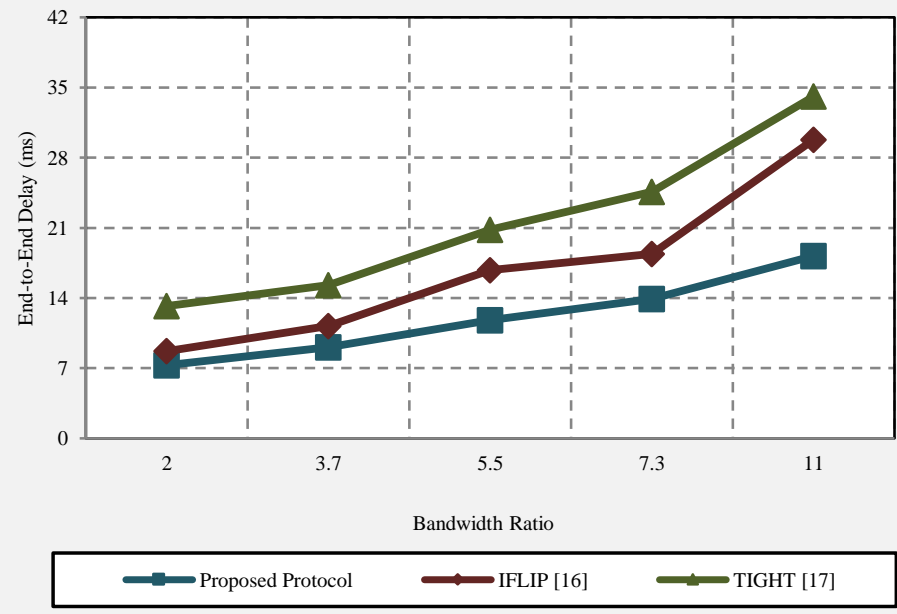

Figure. 6 End-to-end delay over bandwidth ratio

proposed protocol (WPIP) with IFLIP [16] and TIGHT [17] under different bandwidths. In WPIP, $M / G / 1$ queuing model is utilized to avoid the endto-end delay, where IFLIP [16] and TIGHT [17] utilize the first-in-first-out (FIFO) concept in handling the requests and response. FIFO gives better performance in infrastructure based network with limited number of nodes, and it is not sufficient for ad hoc networks like CRAHN. Utilization of $M / G / 1$ queuing model results in reduced queue length and end-to-end delay. Fig. 6 clearly shows that the proposed protocol (WPIP) attains remarkable reduced end-to-end delay for data packet to reach destination than IFLIP [16] and TIGHT [17].

\subsection{Social welfare analysis}

In Fig. 7, percentage of energy saved varying from 0 to 80 are plotted in the $\mathrm{x}$-axis, where the $\mathrm{y}$ axis is plotted with node speed varying from 0 to 10 m/s. Fig. 7, compares WPIP with IFLIP [16] and
TIGHT [17] for social welfare under different node speed. From Fig. 7 It is clearly evident that WPIP saved a remarkable level of energy than IFLIP [16] and TIGHT [17]. When analyzing the reason for the increase in save of energy of all the protocols, it is found that the energy will be consumed more only when the mobility speed is low. Enormous amount of energy is saved is saved by WPIP due to (i) auxiliary and secondary channels, (ii) M/G/1 queuing model, and (iii) available multiple paths. Due to the utilization of (i) auxiliary channels only, (ii) FIFO, and (iii) utilizing single routing path, results reduced energy saving in IFLIP [16] and TIGHT [17].

\section{Conclusion}

Reducing end-to-end delay is a major task in all types of network, because end-to-end delay may lead a network to failure. Solutions for the emerging problems in the real time are available in nature 


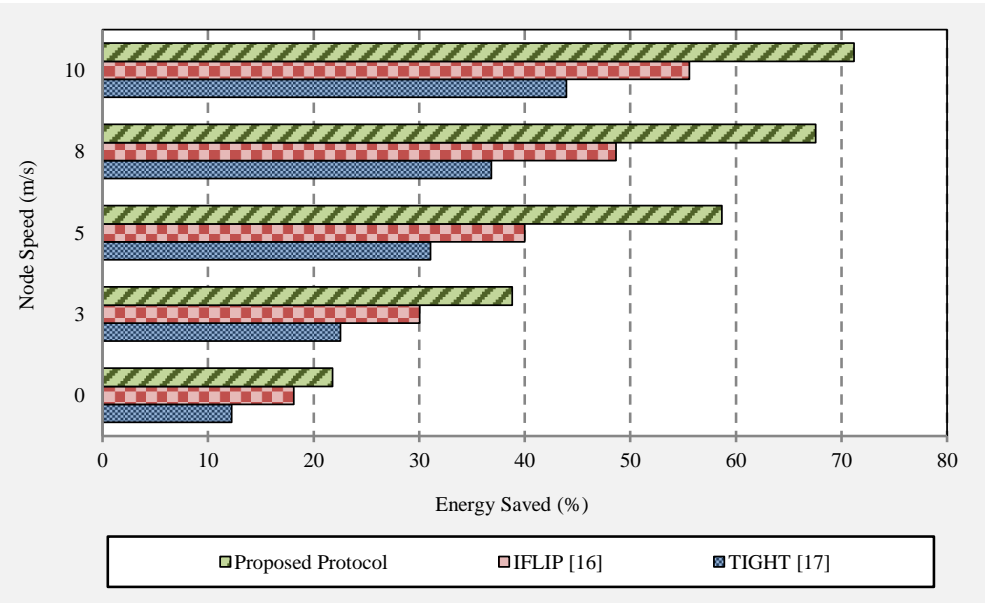

Figure. 7 Social welfare analysis over energy saved

itself. This research work aimed to reduce the endto-end delay in CRAHN by proposing a bio-inspired protocol. The natural characteristics of wolf towards hunting its prey is used in the design of the proposed protocol. When finding the appropriate route to send the received data to the destination, the proposed protocol searches the alternate paths in multiple way. The proposed protocol checks the quality of the paths before sending the data, because sending the data thorough low quality path results in retransmission, waste of energy, and congestion. This research work utilized the $M / G / 1$ queuing model to avoid queue length increased, resulting in avoidance of congestion. NS2 simulator is used to measure the performance of the proposed protocol against the existing protocols with benchmark performance metrics. Results show that the proposed protocol outperforms than the baseline schemes in reducing end-to-end latency and energy utilization. Future dimensions of this research work can be aimed with load balancing resulting to reduce end-to-end latency and energy utilization even more.

\section{References}

[1] J. Mauri, K. Ghafoor, D.B. Rawat, and J.M. Aguiar Perez, "Cognitive Networks: Applications and Deployments", CRC Press, 2015.

[2] M. A. Wyglinski, M. Nekovee, and Y. T. Hou, "Cognitive Radio Communications and Networks Principles and Practice", Academic Press, 2010.

[3] I. F. Akyildiz, W. Y. Lee, M. C. Vuran, and S. Mohanty, "Next Generation/Dynamic Spectrum Access/Cognitive Radio Wireless Networks: A Survey", Computer Networks, Vol.50, pp.2127-2159, 2006.
[4] M. Cesana, F. Cuomo, and E. Ekici, "Routing in Cognitive Radio Networks: Challenges and solutions", Ad Hoc Networks, Vol.9, No.3, pp.228-248, 2011.

[5] K. R. Chowdhury and I. F. Akyildiz, "CRP: A Routing Protocol for Cognitive Radio Ad Hoc Networks", IEEE Journal of Selected Areas in Communications, Vol.29, No.4, pp.794-804, 2011.

[6] S. Basak and T. Acharya, "Cross Layer Optimization for Outage Minimizing Routing in Cognitive Radio Ad Hoc Networks with Primary Users Outage Protection", Journal of Network and Computer Applications, Vol. 98, pp.114-124, 2017.

[7] J. Gao, Y. Zhang, and Y. Liu, "Transport Capacity of Cooperative Cognitive Radio Ad Hoc Networks", Physical Communication, Vol. 25, No. 2, pp.298-303, 2017.

[8] J. S. Singh and M. K. Rai, "CROP: Cognitive Radio Routing Protocol for Link Quality Channel Diverse Cognitive Networks", Journal of Network and Computer Applications, Vol. 104, pp.48-60, 2018.

[9] H. M. A. Abdullah and A. V. S. Kumar, "HFSA-SORA: Hybrid Firefly Simulated Annealing based Spectrum Opportunistic Routing Algorithm for Cognitive Radio Ad hoc Networks (CRAHN)", In: Proc. of 2017 International Conference on Intelligent Computing and Control, pp.1-10, 2017.

[10] N. Mansoor, A. K. M. M. Islam, M. Zareei, and C. Vargas-Rosales, "RARE: A Spectrum Aware Cross-Layer MAC Protocol for Cognitive Radio Ad-Hoc Networks", IEEE Access, Vol. 6, pp.22210-22227, 2018.

[11] F. Tang and J. Li, "Joint Rate Adaptation, Channel Assignment and Routing to Maximize Social Welfare in Multi-Hop Cognitive Radio 
Networks", IEEE Transactions on Wireless Communications, Vol.16, No.4, pp.2097-2110, 2017.

[12] W. Wang, A. Kwasinski, D. Niyato, and Z. Han, "Learning for Robust Routing Based on Stochastic Game in Cognitive Radio Networks", IEEE Transactions on Communications, Vol. 66, No. 6, pp.2588-2602, 2018.

[13] F. Tang, C. Tang, Y. Yang, T. Yang, T. Zhou, J. $\mathrm{Li}$, and M. Guo, "Delay-Minimized Routing in Mobile Cognitive Networks for Time-Critical Applications", IEEE Transactions on Industrial Informatics, Vol.13, No.3, pp.1398-1409, 2017.

[14] R. B. Battula, D. Gopalani, and M. S. Gaur, "Path and Link Aware Routing Algorithm for Cognitive Radio Wireless Mesh Network", Wireless Personal Communications, Vol.96, No.3, pp.3979-3993, 2017.

[15] Z. Che-aron, "An Energy-Efficient and Reliable Routing Protocol for Cognitive Radio Sensor Networks", In: Proc. of International Conference of Advanced Multimedia and Ubiquitous Engineering, Lecture Notes in Electrical Engineering, Vol.448, pp.171-180, 2017.

[16] J. Ramkumar and R. Vadivel, "Improved Frog Leap Inspired Protocol (IFLIP) - for Routing in Cognitive Radio Ad Hoc Networks (CRAHN)", World Journal of Engineering, Vol.15, No.2, pp.306-311, 2018.

[17] X. Jin, R. Zhang, J. Sun, and Y. Zhang, "TIGHT: A Geographic Routing Protocol for Cognitive Radio Mobile Ad Hoc Networks", IEEE Transactions on Wireless Communications, Vol.13, No.8, pp.4670-4681, 2014.

[18] S. Mirjalili, S. M. Mirjalili, and A. Lewis, "Grey Wolf Optimizer", Advances in Engineering Software, Vol.69, pp.46-61, 2014.

[19] O. C. Ibe, Markov Processes for Stochastic Modeling (Second Edition), Chp. 7, Elsevier, 2013. 